\title{
CT IMAGING FEATURES OF PRIMARY PERITONEAL TUMORS WITH PATHOLOGICAL CORRELATION
}

\section{DR RASHMI CHAND, DR SAUGATA SEN AND DR DIVYA MIDHA- TATA MEDICAL CENTER, KOLKATA, INDIA}

The purpose of the exhibit is to review the CT imaging patterns of primary peritoneal tumors and correlate with the pathological findings. Primary peritoneal tumors are defined as tumors with primary manifestation in the peritoneum in the absence of a visceral site of origin .The peritoneum is a thin, translucent serosal membrane of mesodermal origin. They arise from Mesothelial, Sub mesothelia, Mesenchymal and Uncommitted stem cells.

Based on the proposed histogenesis, they are classified into :1. Mesothelial 2. Epithelial 3. Smooth muscle 4. Uncertain origin groups MESOTHELIAL ORIGIN 1: Peritoneal malignant mesothelioma- Diffuse and localized 2. Cystic mesothelioma3. Papillary 4. Adenomatoid

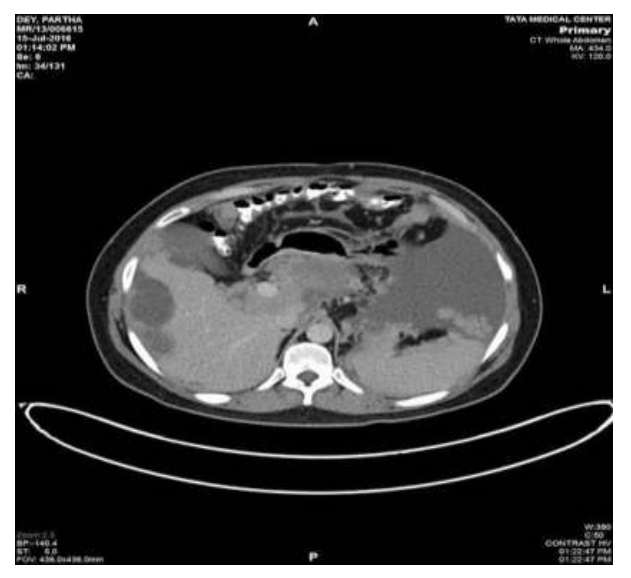

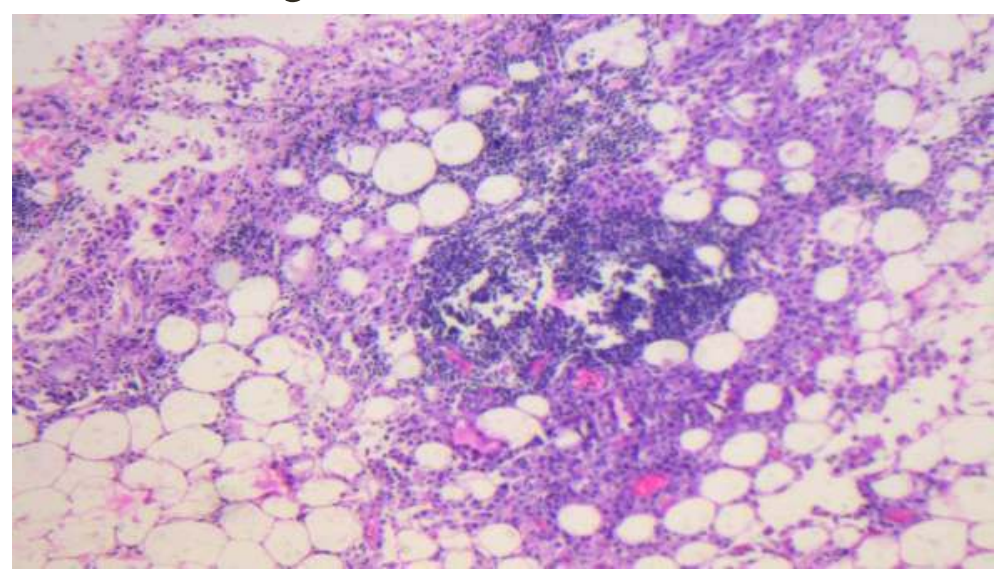

Diffuse Mesothelioma

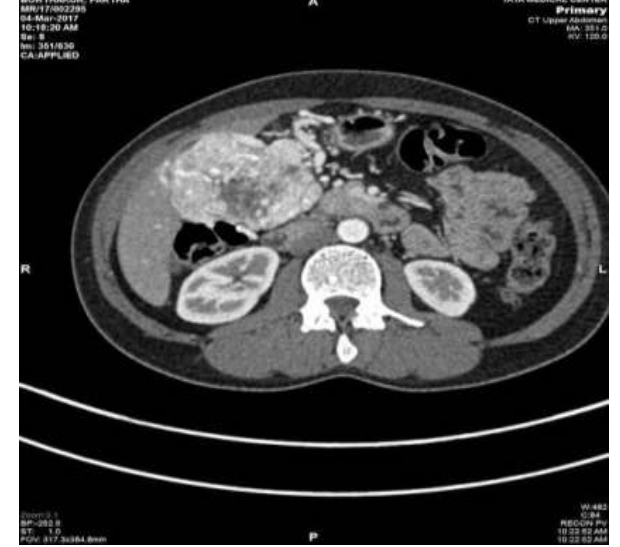

Localised Mesothelioma

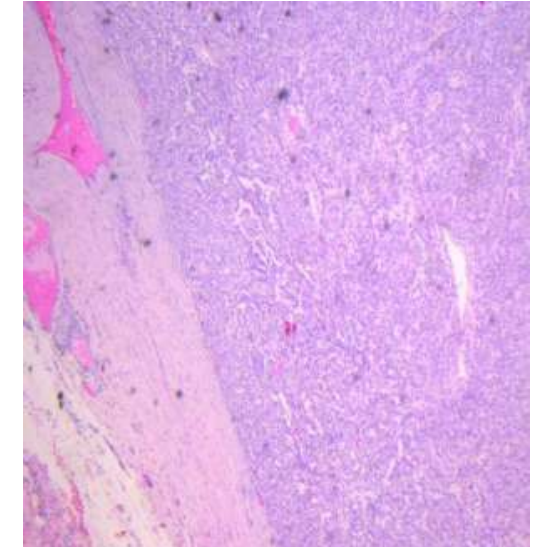

Invasion of GB serosa with multiple serosal deposits.

EPITHELIAL ORIGIN - 1. Primary peritoneal serous carcinoma 2. Primary Peritoneal serous borderline tumor

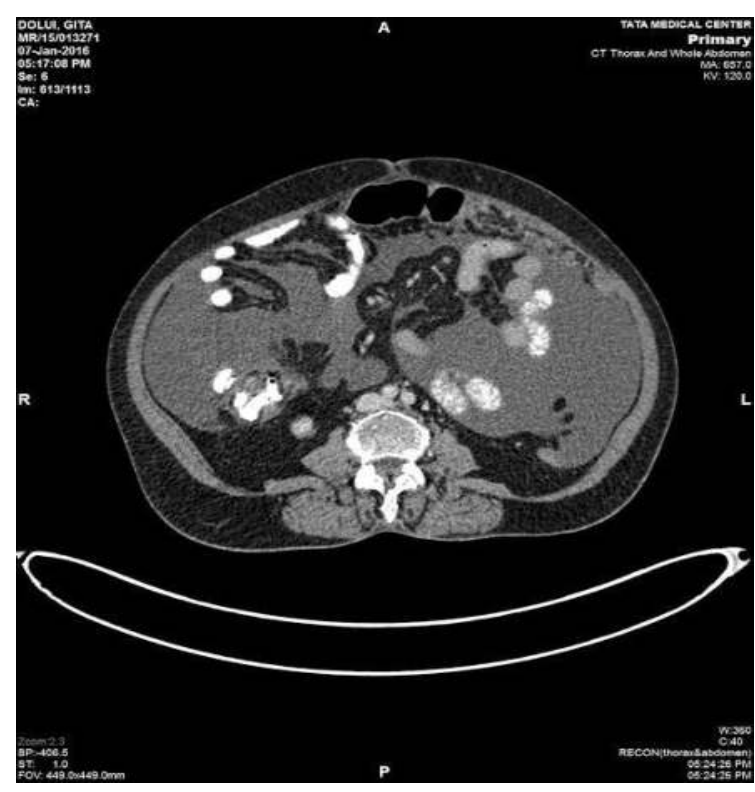

Primary Peritoneal Serous Carcinoma

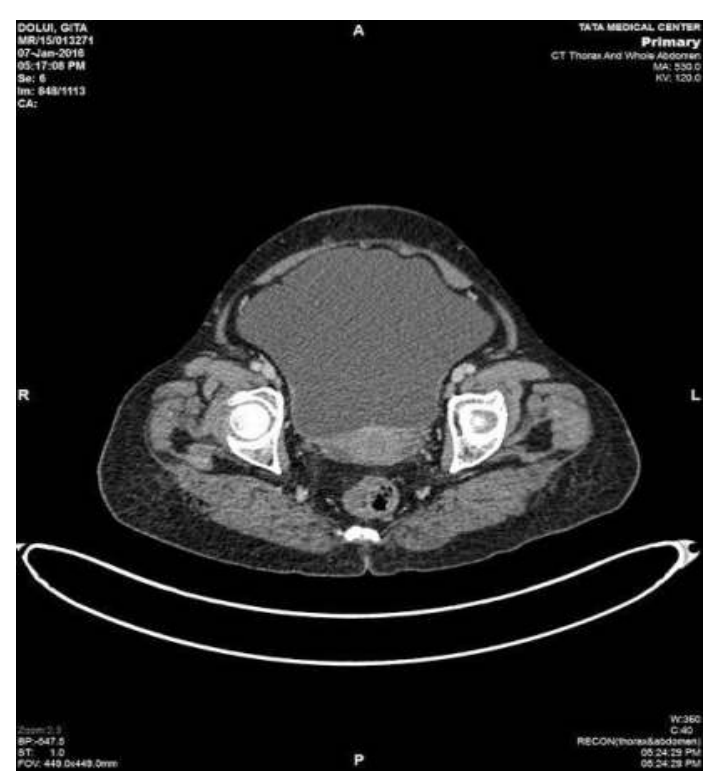

Normal Ovaries

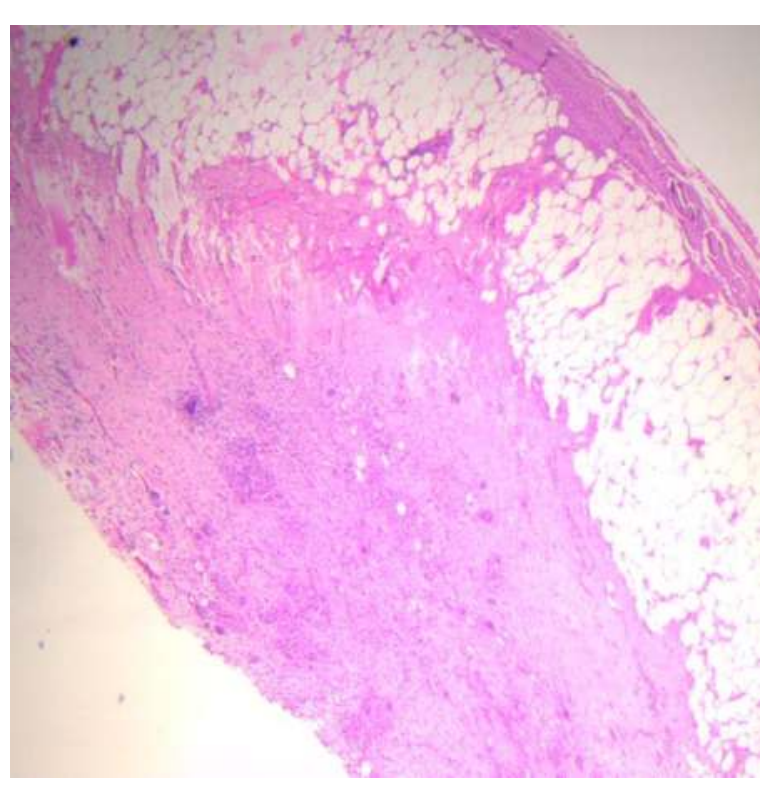

Normal Serosa of ovaries

SMOOTH MUSCLE TUMOR - 1 Leiomyomatosis peritonealis disseminate (LPD)

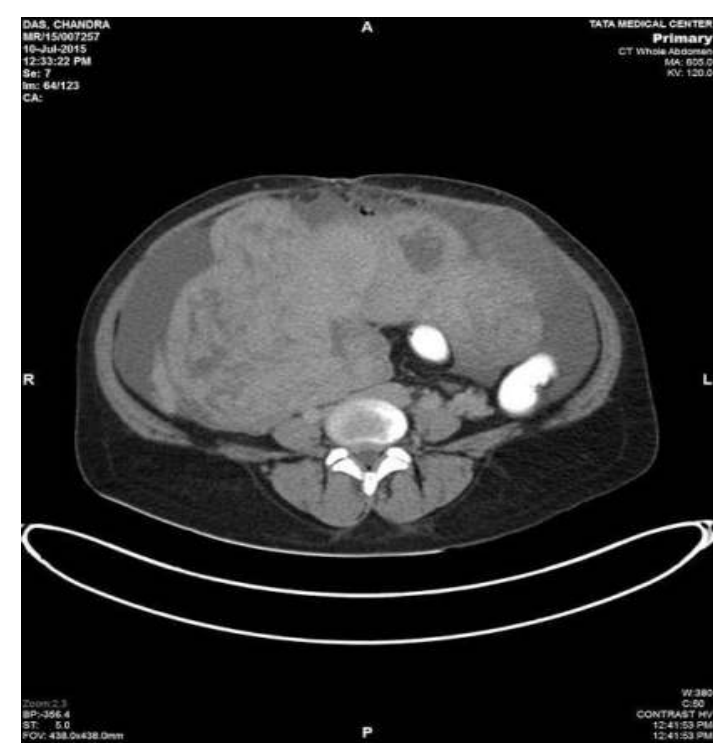

LPD - Multiple Enhancing deposits throughout peritoneum and Pelvis with Ascites

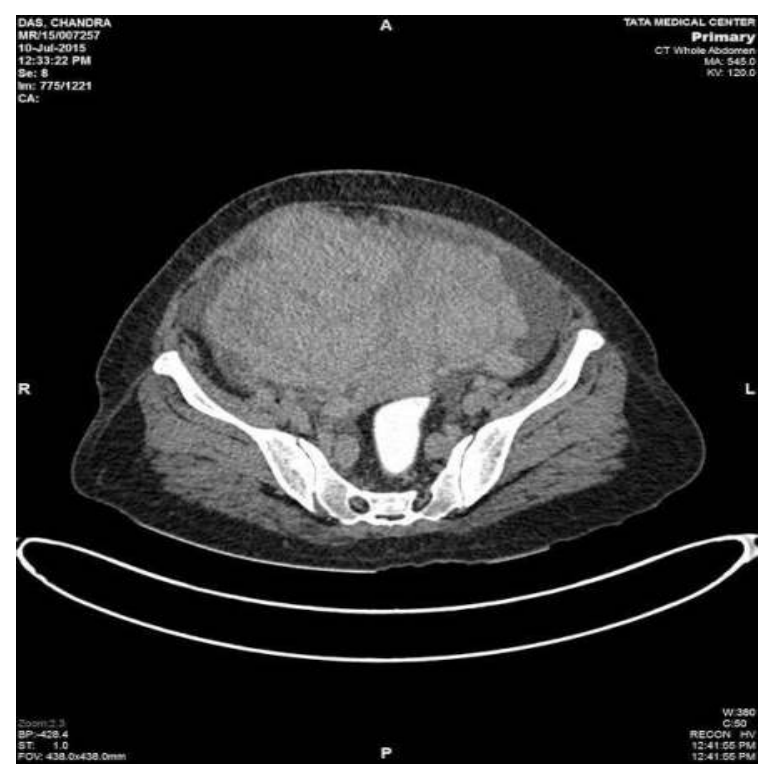

Fascicles of benign smooth muscle cells

TUMORS OF UNKNOWN ORIGIN - 1. Desmoplastic small round cell tumor (DSRC) 2. Solitary fibrous tumor (SFT)

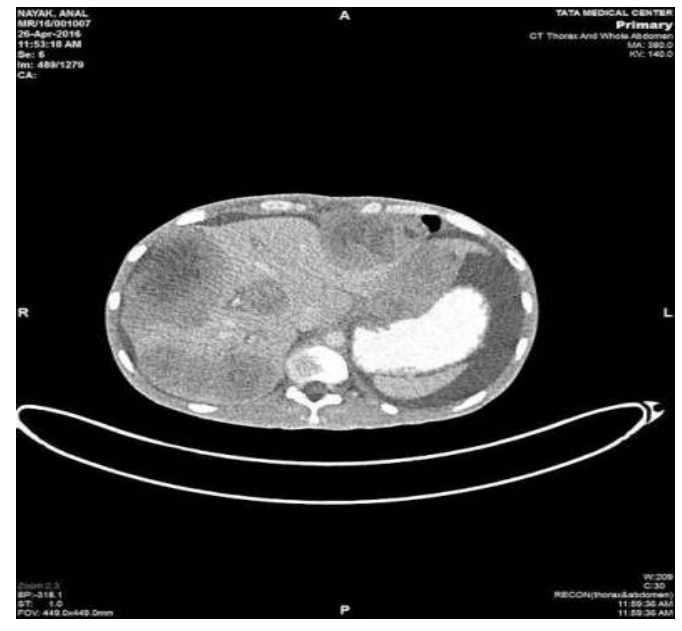

DSRC with peritoneal deposits

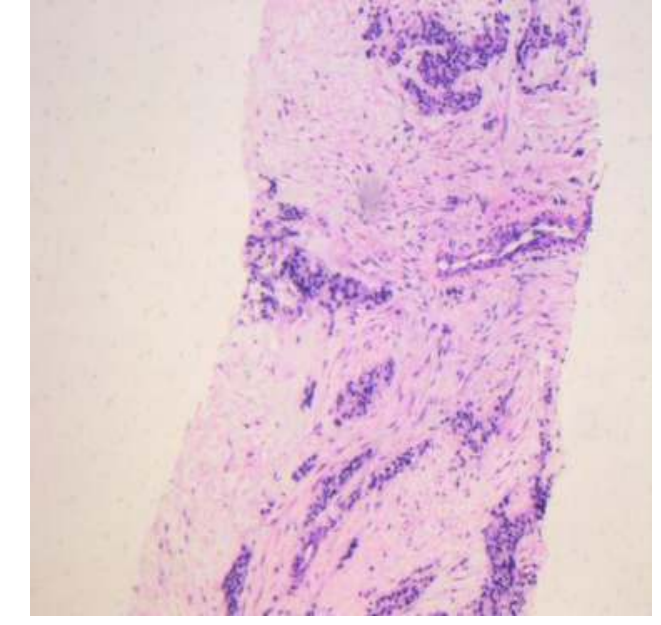

DSRC with small round cells

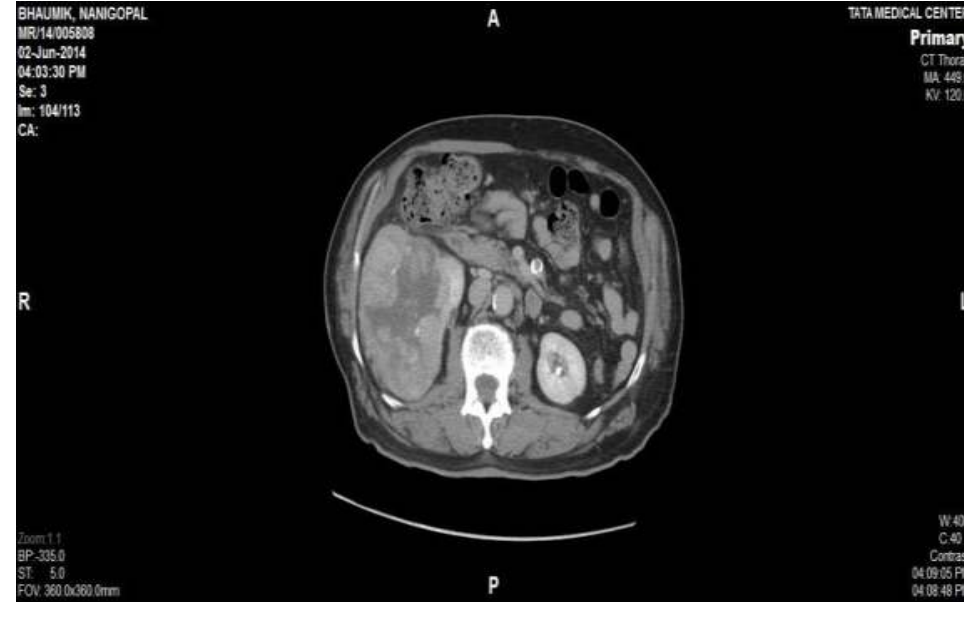

SFT with degeneration

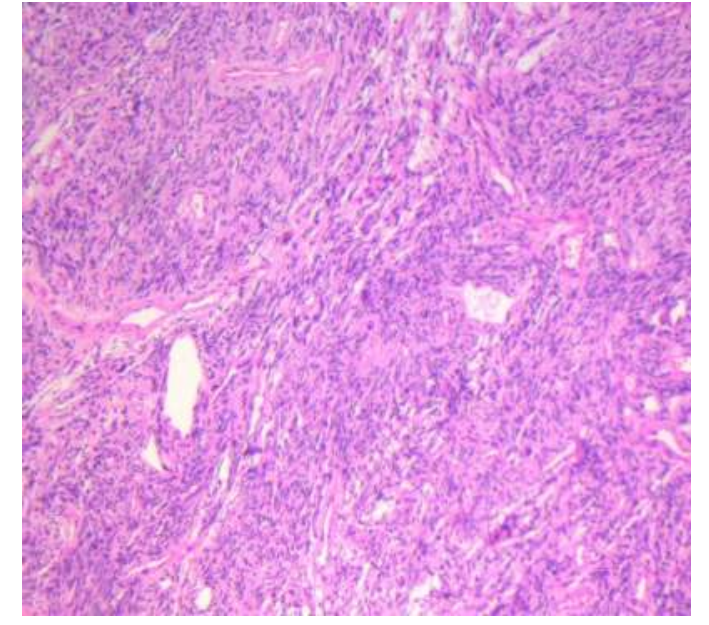

SFT with spindle cells \& Collagen

CONCLUSION : Imaging appearances of primary peritoneal tumors overlap yet are distinctly different clinically. Hence differentiating from metastatic disease is important for appropriate management. 